with transmission electron microscopy, the researchers conclude that the GNF cathode consisted of large numbers of fibers that were about $50 \mathrm{~nm}$ in diameter but were not carbon nanotubes. Electrons were drawn from the cold GNF cathode through field emission; that is, a strong electric field between the GNF cathode and the $\mathrm{Cu}$ anode pulled the electrons off. The researchers, T. Matsumoto of Stanley Electric Corp. in Yokohama, Japan, and H. Mimura of Tohoku University in Sendai, Japan, chose a simple diode configuration (instead of the usual triode), not only for its superior $x$-ray generation efficiency but also for its potential advantages in size and cost. To assure that the $\mathrm{x}$-rays would emerge from a point, they restricted the electron bombardment region of the $\mathrm{Cu}$ anode by coating all but about $10 \mu \mathrm{m}$ of the sharpened metal tip with an insulating silicate.

Mastumoto and Mimura investigated the current-voltage characteristics of the efficient GNF cathode and also spectrally characterized the point source's x-ray output using a Ge detector and a multichannel analyzer. Next, they imaged a tungsten mesh and $\mathrm{a} \mathrm{Pb}$ x-ray test chart in the transmission geometry, where the test object is between the x-ray source and the detector (a charge-coupled device camera with a NaI scintillator). The system achieved a high resolution, roughly $10 \mu \mathrm{m}$, with an image acquisition time of $10 \mathrm{~s}$ using a charge-coupled device.

"There is great interest in building a practical, efficient, high-current-density x-ray device," said Matsumoto, "and we've now developed a simple $x$-ray radiography system. Since the efficient field-emission process eliminates the usual bulky water-cooling system, this makes possible a portable, compact $\mathrm{x}$-ray system."

RichARD LOUIE

\section{Incorporation of Particle Size Distribution Simplifies Modeling of SiCN Powder Oxidation Kinetics}

The oxidation kinetics of polymerderived ceramic materials is often studied using powdered samples because of the difficulty in obtaining dense specimens. The change in sample mass is measured as a function of time, and the data is fitted to a parabolic oxidation model. To simplify data analysis, the powder sample is treated as an assembly of perfectly spherical particles with identical radii. The monosize model results in significant errors in the value of the rate constant obtained from the measurement. Recently, R. Raj, A. Saha, and S. Shah of the Department of Mechanical Engineering at the University of Colorado, Boulder, have developed a model that accounts for the particle size distribution in the powder. Application of this statistical model to the time-dependent oxidation of silicon carbonitride ( $\mathrm{SiCN})$ yielded a much more accurate value of the rate constant than that provided by the monosize model. According to Raj, "This improved data analysis technique gives much greater credibility to the simple experimental approach of using powders to study material oxidation rates."

As described in the February issue of the Journal of the American Ceramic Society, the researchers prepared SiCN powder by cross-linking and pyrolyzing Ceraset-SN, a commercially available precursor. The final product was crushed into powder and imaged using scanning electron microscopy. Size measurements from the micrographs were used to generate a histogram of radius values and an average radius value. The $\mathrm{SiCN}$ was oxidized in air at $1350^{\circ} \mathrm{C}$ for $150 \mathrm{~h}$, with the mass of the sample recorded periodically throughout the process. The monosize and statistical models were then fitted to this data.

Data analysis with the monosize model yielded two limiting values for the oxidation rate constant because one rate constant was needed to fit the data for short-term oxidation kinetics and the other for long-term oxidation. In this study, this parabolic rate constant $k_{\mathrm{p}}$ was found to lie in the range $19.7 \times 10^{-18} \mathrm{~m}^{2} / \mathrm{s}<$ $k_{\mathrm{p}}<2.7 \times 10^{-18} \mathrm{~m}^{2} / \mathrm{s}$. The statistical analysis, on the other hand, resulted in a theoretical curve that fit the entire time span of the data, using only one value of the rate constant $4.5 \times 10^{-18} \mathrm{~m}^{2} / \mathrm{s}$ in this study, which greatly improved the accuracy of rate constant determination.

Raj is currently developing a statistical model that will account for the distribution in the particle shape as well as size. Current models assume a spherical particle shape.

GREG KHITROV

\section{Emitting Amorphous Molecular Materials for} Electroluminescence Designed

Recently, amorphous molecular materials have received a great deal of attention as a new class of functional organic materials because of their isotropic properties, due to the absence of grain boundaries, and ease of uniform film formation. In the

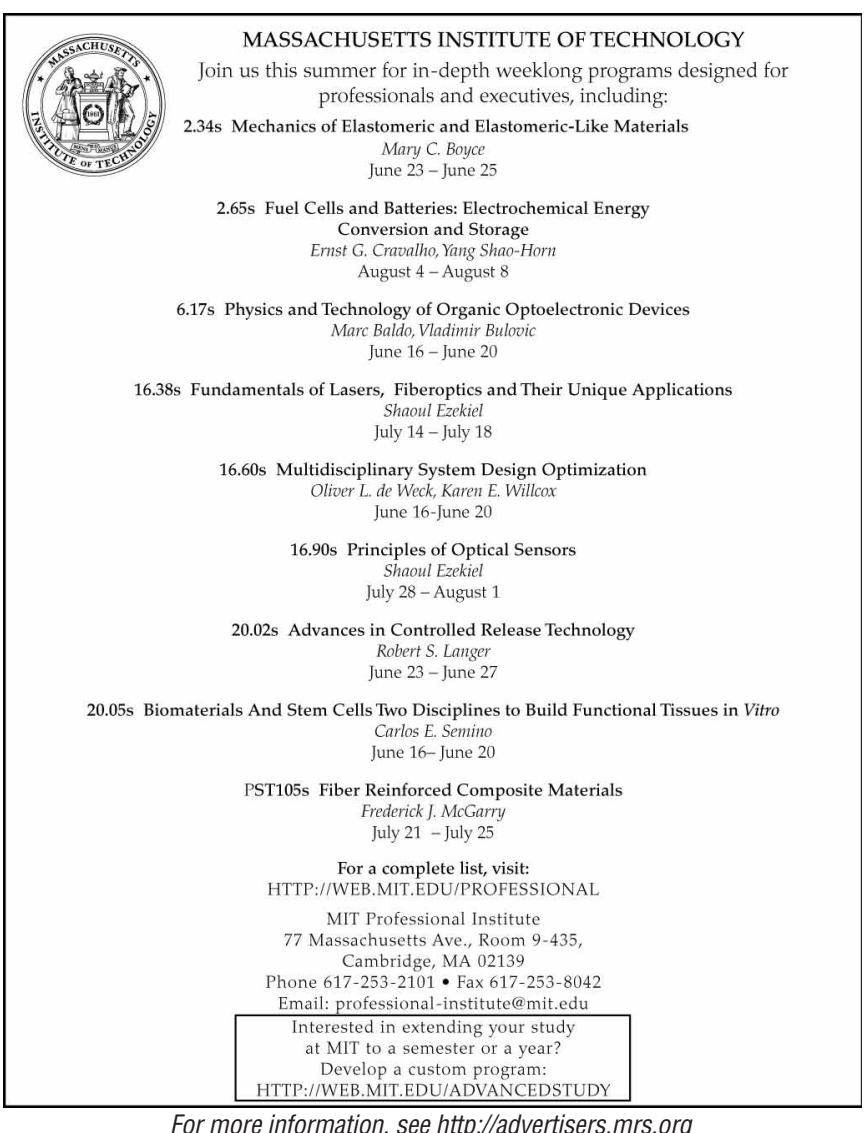


March 11 issue of Chemistry of Materials, Y. Shirota and co-workers from Osaka University in Japan reported the synthesis and properties of a novel class of highperformance, color-tunable emitting amorphous molecular materials with bipolar characters: 4-dimesitylboryl-N,N-bis(9,9dimethylfluoren-2-yl)aniline (FlAMB-0T); $2-\{4$ - [bis- $(9,9$-dimethylfluoren-2yl)amino]phenyl\}-5-(dimesitylboryl) thiophene (FlAMB-1T); 2-\{4-[bis- $(9,9-$ dimethylfluoren-2-yl)amino]phenyl $\}-2^{\prime}-$ dimesitylboryl-5,5'-bithiophene (FlAMB$2 \mathrm{~T})$; and 5-\{4-[bis $(9,9$-dimethylfluoren-2yl)amino]phenyl -5"-dimesitylboryl2,2':5',2"'-terthiophene (FlAMB-3T).

The molecular design of these materials is based on connecting both electrondonating and electron-accepting moieties (which undergo reversible oxidation and reduction) through a central $\pi$-conjugated system. In other words, the incorporation of the difluorenyl(phenyl)amine and dimesitylboron moieties provide both electron-donating and electron-accepting properties, respectively, and facilitated formation of amorphous glasses due to their nonplanar molecular structures. The central thiophene rings control the highest occupied and lowest unoccupied molecu- lar orbital energy levels, depending on the material's $\pi$-conjugation length. The researchers reported that emission color can be tuned by varying the conjugation length of the thiophene unit.

The researchers reported the fabrication of electroluminescent devices using FlAMB$n \mathrm{~T}$ as emitting or host materials and the development of a high-performance whitelight-emitting electroluminescent device using a bilayer combination of FlAMB-0T and FlAMB-3T.

ANDREI A. ELISEEV

\section{Surface Properties Reversibly Switched Using Electrical Potential}

A research team from the Massachusetts Institute of Technology (MIT), the University of California at Santa Barbara (UCSB), and UC-Berkeley have developed a process that dynamically changes interfacial properties by conformational transitions, or switching, of surface-confined molecules. Unlike other methods that involve chemical reactions, the team has demonstrated the ability to use an active stimulus, such as an electrical potential, to effect such changes without altering the system's environment.

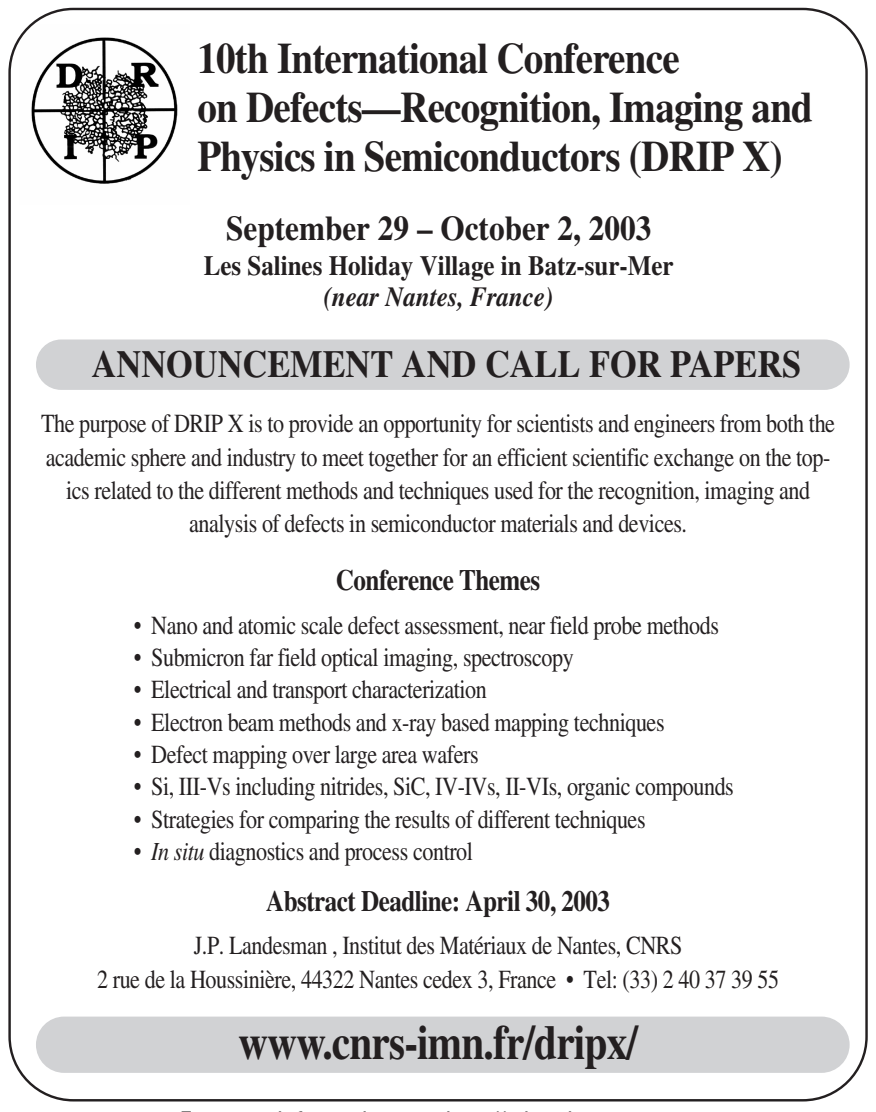

For more information, see http://advertisers.mrs.org
As reported in the January 17 issue of Science, a self-assembled monolayer of (16-mercapto)hexadecanoic acid was prepared on a gold surface. This molecule was chosen because it has a hydrophobic chain and a hydrophilic group on one end; therefore, it may grant different properties to the surface, depending on which part of the molecule is exposed. In order to allow for the switching, the monolayers need to be less dense than conventional self-assembled monolayers; that is, sufficient spatial freedom must be established for each molecule. A monolayer with an optimum spacing between molecules was obtained using a precursor molecule with a globular head group. Hydrolysis of the globular group yielded a low-density monolayer of the molecule of interest.

In the equilibrium state, the molecules organized in a straight fashion on the surface, exposing their hydrophilic end. Joerg Lahann of MIT, Samir Mitragotri of UCSB, and co-workers observed that after applying an electrical potential to the gold surface, the molecules' end groups were attracted toward the charged surface, showing the hydrophilic chains. Sum frequency generation (SFG) spectroscopy was used to analyze the conformational states of the monolayer, since the intensity of the SFG spectrum is affected by the orientation of the molecules. The results show that an electrical potential changes the conformation of the molecules in the monolayer, and that the switching is a reversible process. Contact angles were measured over four switching cycles, and while a large hysteresis was observed, the drop in contact angle was also determined to be reversible. The researchers attributed the observed hysteresis to surface chemical heterogeneity and roughness.

According to the researchers, these findings have important implications for the dynamic control of macroscopic surface properties. The researchers identified applications in microfluidics, microengineering of smart templates, and microfabrication of controlled-release devices.

Maria M. CORTALEZZI

\section{Stable $\alpha$-Phase Nickel Hydroxides Obtained with $10 \%$ Aluminum Substitution}

Alpha-phase nickel hydroxides have recently been investigated in order to increase the performance of rechargeable alkaline batteries. The $\alpha$-nickel hydroxide has a superior electrochemical capacity of $433 \mathrm{mAh} / \mathrm{g}$ theoretical and $350 \mathrm{mAh} / \mathrm{g}$ actual, as compared with $\beta$-nickel hydroxide at $289 \mathrm{mAh} / \mathrm{g}$ theoretical and 273 